\title{
Idiopathic Granulomatous Hypophysitis: A Case Report and Literature Review
}

\author{
Tae Oong Eom, Eun Suk Park, Sung Ho Park, Min Soo Kim, Soon Chan Kwon, Hong Bo Sim, \\ In Uk Lyo, Jun Bum Park
}

Department of Neurosurgery, Ulsan University Hospital, University of Ulsan College of Medicine, Ulsan, Republic of Korea

Corresponding author:

Eun Suk Park

Department of Neurosurgery, Ulsan University Hospital, University of Ulsan College of Medicine, 877, Bangeojinsunhwando-ro, Dong-gu, Ulsan 44033, Republic of Korea

Tel: +82-52-250-7139

Fax: +82-52-250-7138

E-mail: esparkns@naver.com,

espark@uuh.ulsan.kr

Received: May 1, 2018

Revised: June 26, 2018

Accepted: June 28, 2018
Idiopathic granulomatous hypophysitis (IGH) is a rare inflammatory disorder of the pituitary that causes transient or permanent gland dysfunction and various neurological symptoms. We present a case involving a 24-year-old woman who was admitted for headache and visual disturbance. The patient was preoperatively diagnosed with pituitary adenoma and underwent transsphenoidal surgery. Histopathology revealed a diagnosis of granulomatous hypophysitis. She was finally diagnosed with IGH given the absence of systemic granulomatous disease. Because IGH is a rare disease and is easily misdiagnosed as other pituitary disease, careful inspection of patient symptoms is required. Surgical intervention provides a histological diagnosis and immediate mass reduction in patients with $\mathrm{IGH}$; however, hormone replacement is frequently required, and long-term follow up is very important.

Key Words: Hypophysitis; Pituitary diseases; Pituitary gland; Sella turcica

\section{INTRODUCTION}

Hypophysitis is a relatively rare disorder caused by inflammatory infiltration of the pituitary gland that can cause tissue destruction, transient or permanent gland dysfunction, and endocrinopathies $^{3-5)}$. Hypophysitis has 4 histopathological subtypes: lymphocytic, granulomatous, xanthomatous, and necrotizing ${ }^{16)}$. Among these, granulomatous hypophysitis is the second most common subtype and features discrete granulomas of multinucleated giant cells and histiocytes with surrounding lymphocytes and plasma cells ${ }^{8,25}$. Granulomatous hypophysitis can occur as a primary phenomenon or secondary to systemic disease. Secondary causes of hypophysitis include systemic disease (e.g., sarcoidosis, Langerhans cell histiocytosis, Wegener's granulomatosis, disseminated tuberculosis, and syphilis), an underlying pituitary lesion (e.g., Rathke cleft cyst and adenoma), and certain medications (e.g., ipilimumab) ${ }^{16,27)}$. A diagnosis of idiopathic granulomatous hypophysitis (IGH) is made when no other inflammatory cause can be identified. Here, we report a case of histologically confirmed IGH involving sellar mass lesions and pituitary dysfunction.

\section{CASE REPORT}

A 24-year-old woman presented with complaints of blurred vision and headache. On physical examination, the visual fields, fundoscopy findings, and neurological examination results were unremarkable. Endocrinological evaluation revealed hyperprolactinemia of $34.3 \mathrm{ng} / \mathrm{mL}$ (normal, 5.3-22.2) and otherwise nonspecific findings. Magnetic resonance imaging (MRI) showed a symmetric sellar mass that was $17 \mathrm{~mm}$ in size and produced mild upward displacement of the optic chiasm. The mass was isointense with gray matter on T1-weighted images (WI) and T2-WI and exhibited homogeneous gadolinium enhancement (Fig. 1). Accordingly, the patient was initially diagnosed with pituitary adenoma and secondary hyperprolactinemia. The patient underwent surgical exploration via a transsphenoidal approach. Intraoperative examination revealed that the whole pituitary fossa was occupied by a fibrous yellowish mass that adhered to the adjacent dural mater. It didn't have a clear cleavage between inflammation and the surrounding pituitary gland. Therefore, only a partial resection was achieved under neuronavigation guidance. Histological examination revealed a non-necrotizing granulomatous lesion with chronic inflammation (Fig. 2). After histological diagnosis of the lesion as granulomatous hypophysitis, the patient further underwent cutaneous, skeletal, visceral, and laboratory examinations to exclude systemic granulomatous disease. Additional examinations included interferon- $\gamma$ release assays (IGRA) for tuberculosis, antineutrophil cytoplasmic antibody and anti-nuclear antibody for vasculitis, angiotensin converting enzyme (ACE) and chest computed tomography (CT) for sarcoidosis. Given the absence of 

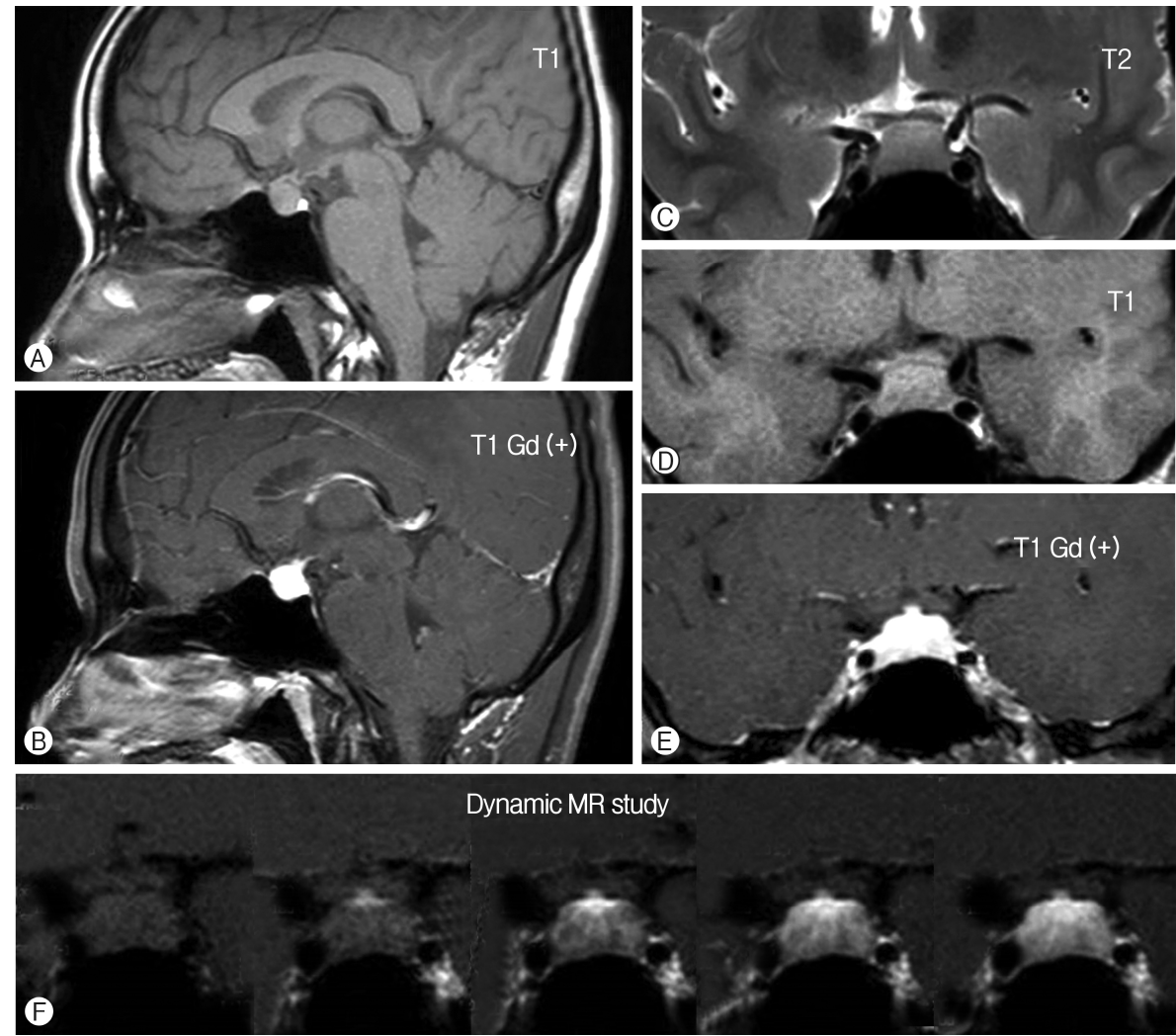

Dynamic MR study
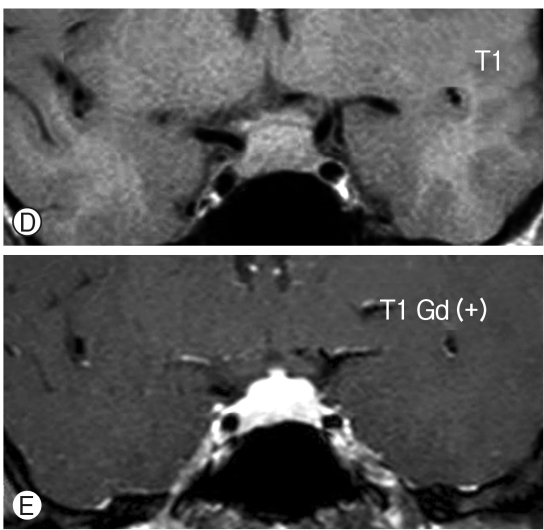

Fig. 1. A 24-year-old woman with previous no medical history presented with blurred vision and headache. Brain magnetic resonance imaging (MRI) showed a $1.7 \mathrm{~cm}$ sized sellar mass with contacting and mildly displacing the optic chiasm. The mass showed isointense on $\mathrm{T} 1$-weighted and T2-weighted images and exhibited homogeneous gadolinium (Gd) enhancement. The patient was initially diagnosed with pituitary adenoma. (A) T1-weighted sagittal image, (B) Gd-enhanced T1-weighted sagittal image, (C) T2-weighted coronal image, (D) T1-weighted coronal image, (E) Gd-enhanced T1-weighted coronal image, and (F) dynamic Gd-enhanced MRI.

systemic disease, the patient was finally diagnosed with IGH.

The postoperative course was uneventful. The patient's symptoms gradually resolved and steroid treatment was gradually stopped. Although the surgical resection was limited, MRI at 6 months follow-up revealed a marked reduction in size of the sellar mass and dramatic resolution of the pituitary lesion (Fig. 3).

\section{DISCUSSION}

IGH is a rare chronic inflammatory disorder of the pituitary and accounts for less than $1 \%$ of all pituitary disorders ${ }^{22}$. Although it is generally thought that IGH has no sex predilection $^{2,19)}$, Su et al. ${ }^{24)}$ reviewed 38 cases of IGH and reported female predilection with an approximate ratio of 2:1 (26:12). A recent systematic review of 82 cases found that $72 \%$ of patients were women $^{14)}$. In these studies, the mean ages of presentation were 46.1 years and 44.4 years, respectively ${ }^{14,24)}$. The clinical presentation of IGH is usually that of an expanding pituitary

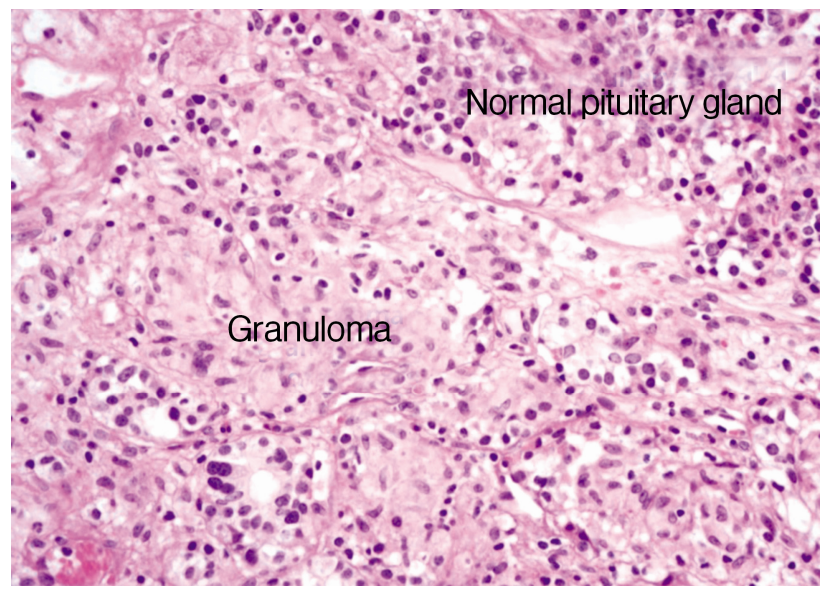

Fig. 2. Histological examination revealed a non-necrotizing granulomatous lesion with chronic inflammation. Hematoxylin and eosinstained $(H \& E)$ sections from the biopsy revealed focal replacement of pituitary architecture by a lymphocytic infiltrate, and noncaseating epithelioid cell granulomas with multinucleated giant cells and areas of fibrosis (H \& E stain, $\times 400$ magnification). 

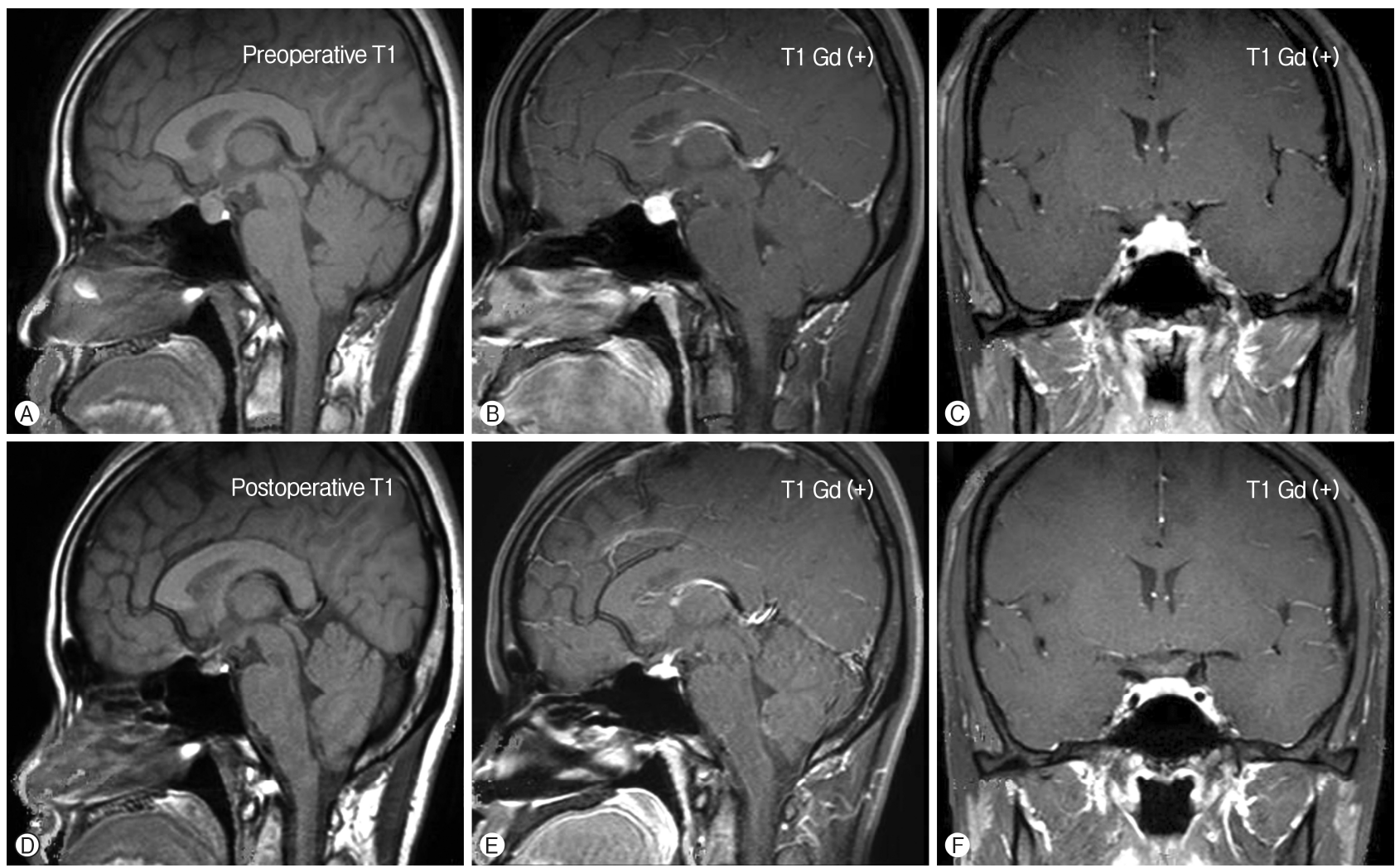

Fig. 3. Intraoperative findings revealed inflammatory sellar mass, which didn't have a clear cleavage with the surrounding pituitary gland. Therefore, a partial resection was achieved under neuronavigation guidance. The postoperative magnetic resonance imaging (MRI) at 6 months follow-up revealed a marked reduction in size of the sellar mass and dramatic resolution of the pituitary lesion compared to preoperative MRI. (A) Preoperative T1-weighted sagittal image, (B) preoperative gadolinium (Gd)-enhanced T1-weighted sagittal image, (C) preoperative Gd-enhanced T1-weighted coronal image, (D) postoperative T1-weighted sagittal image, (E) postoperative Gd-enhanced T1weighted sagittal image, and (F) postoperative Gd-enhanced T1-weighted coronal image.

mass lesion with insidious onset and a progressive course. The most common presenting symptom of IGH in the literature was headache, followed by visual disturbances and symptoms related to diabetes insipidus. Other symptoms included vomiting, nausea, extraocular muscle paralysis, fever, and galactorrhea. Given an insidious clinical course mimicking pituitary adenoma and acute progress mimicking acute meningitis ${ }^{1,7,26,29)}$, it is difficult to correctly diagnose IGH on admission.

IGH typically presents with diffuse enlargement of the pituitary mass and enlargement of the pituitary stalk. At present, MRI is the most useful tool for diagnosing IGH and has distinct advantages over CT for imaging pituitary and sellar regional lesions. Based on a review by Su et al. ${ }^{24)}$, MRI findings of primary hypophysitis include: (1) diffuse, ill-defined, symmetrical enlargement of the pituitary tissue; (2) thickening of the pituitary stalk without deviation or impaired visualization; (3) an intact sellar floor; (4) isointensity with the surrounding gray matter on T1-WI and marked homogeneous or heterogeneous enhancement by gadolinium with a strip of enhanced tissue along the dura mater (the so-called "dural tail"); and (5) delayed complete contrast enhancement of the whole pituitary on dynamic
MRI (>90 s) ${ }^{1,6,11,13,18,20,21,24,28)}$. Among these MRI findings, the best predictors of primary hypophysitis are diffuse pituitary enlargement, marked enhancement of the pituitary mass, and pituitary stalk thickening ${ }^{24)}$.

A diagnosis of IGH should exclude secondary granulomatous hypophysitis. The etiology of secondary granulomatous hypophysitis includes infection (e.g., tuberculosis, syphilis, and fungal infection), systemic inflammatory conditions (e.g., sarcoidosis, Wegener's granulomatosis, Takayasu's arteritis, Crohn's disease, and histiocytosis X), and foreign body reactions (e.g., ruptured Rathke's cyst and mucocele $)^{15)}$. Despite diagnostic advances, the differentiation of primary and secondary cases of granulomatous hypophysitis remains dificult. In cases where granulomatous hypophysitis is suspected, all available steps should be taken to exclude possible etiologies of infection or systemic disease, including Mantoux testing, chest radiography, tuberculosis PCR of cerebrospinal fluid and pituitary tissue, serum syphilis testing, and serum ACE measurement.

The possible etiology of IGH is not well understood and the first-line treatment is controversial. Some studies have reported satisfactory responses to high-dose steroid therapy ${ }^{14,15,17)}$. 
Conservative management with close clinical observation has also been advocated as a therapeutic option given the often benign and transient course of the disease and occasional spontaneous remission". Yet, transsphenoidal surgery has both diagnostic and therapeutic utility, and should be performed in cases of progressive compression, and especially in cases of atypical clinical or radiological presentation or an unconfirmed diagnosis. Additionally, a majority of reported IGH cases and approximately half of primary hypophysitis cases were preoperatively misdiagnosed as pituitary adenomas in previous reports, similar to our case ${ }^{10,17,23)}$. Surgery provides tissue for histological diagnosis and allows rapid decompression of the mass lesion, thereby resolving headache and visual defects ${ }^{12,13)}$. Furthermore, surgery and definitive histological diagnosis can prevent the unnecessary use of high-dose steroid therapy and facilitate the appropriate treatment of other conditions such as infection or neoplasm. Nonetheless, one study reported no significant difference in the outcomes of patients who underwent surgical excision versus those who underwent pituitary biopsy and corticosteroid treatment ${ }^{14)}$. These are all important issues for consideration when diagnosing and treating patients with IGH.

\section{CONCLUSION}

IGH is a rare inflammatory disease of unknown etiology that is easily misdiagnosed as pituitary adenoma or pituitary infection. The outcome of surgery for IGH is favorable, providing a histological diagnosis and immediate mass reduction, but hormone replacement is frequently required and long-term follow-up is very important.

\section{ACKNOWLEDGMENT}

This work was supported by the National Research Foundation of Korea (NRF) grant funded by the Korea government (MSIT) (No. 2017R1C1B5018208).

\section{CONFLICTS OF INTEREST}

No potential conflict of interest relevant to this article was reported.

\section{REFERENCES}

1. Bhansali A, Velayutham P, Radotra BD, Pathak A: Idiopathic granulomatous hypophysitis presenting as non-functioning pituitary adenoma: description of six cases and review of literature. Br J Neurosurg 18:489-494, 2004

2. Brisman MH, Morgello S, Silvers A, Klein I, Post KD: Idiopathic granulomatous hypophysitis. Neurosurg Focus 1:e7, 1996
3. Buxton N, Robertson I: Lymphocytic and granulocytic hypophysitis: a single centre experience. Br J Neurosurg 15:242-245, discussion 245-246, 2001

4. Caturegli P, Lupi I, Landek-Salgado M, Kimura H, Rose NR: Pituitary autoimmunity: 30 years later. Autoimmun Rev 7:631637, 2008

5. Caturegli P, Newschaffer C, Olivi A, Pomper MG, Burger PC, Rose NR: Autoimmune hypophysitis. Endocr Rev 26:599-614, 2005

6. Cheung CC, Ezzat S, Smyth HS, Asa SL: The spectrum and significance of primary hypophysitis. J Clin Endocrinol Metab 86: 1048-1053, 2001

7. Cooper R, Belilos E, Drexler S, Efron A, Ferrara E, Tollin SR: Idiopathic giant-cell granulomatous hypophysitis mimicking acute meningitis. Am J Med Sci 318:339-342, 1999

8. Doniach I, Wright EA: Two cases of giant-cell granuloma of the pituitary gland. J Pathol Bacteriol 63:69-79, 1951

9. Ezzat S, Josse RG: Autoimmune hypophysitis. Trends Endocrinol Metab 8:74-80, 1997

10. Flanagan DE, Ibrahim AE, Ellison DW, Armitage M, GawneCain M, Lees PD: Inflammatory hypophysitis - the spectrum of disease. Acta Neurochir (Wien) 144:47-56, 2002

11. Gazioğlu N, Tüzgen S, Oz B, Koçer N, Kafadar A, Akar Z, et al.: Idiopathic granulomatous hypophysitis: are there reliable, constant radiological and clinical diagnostic criterias? Neuroradiology 42:890-894, 2000

12. Higuchi M, Arita N, Mori S, Satoh B, Mori H, Hayakawa T: Pituitary granuloma and chronic inflammation of hypophysis: clinical and immunohistochemical studies. Acta Neurochir (Wien) 121:152-158, 1993

13. Honegger J, Fahlbusch R, Bornemann A, Hensen J, Buchfelder M, Muller M, et al.: Lymphocytic and granulomatous hypophysitis: experience with nine cases. Neurosurgery 40:713-722; discussion 722-713, 1997

14. Hunn BH, Martin WG, Simpson S, Jr., McLean CA: Idiopathic granulomatous hypophysitis: a systematic review of 82 cases in the literature. Pituitary 17:357-365, 2014

15. Imber BS, Lee HS, Kunwar S, Blevins LS, Aghi MK: Hypophysitis: a single-center case series. Pituitary 18:630-641, 2015

16. Leporati P, Landek-Salgado MA, Lupi I, Chiovato L, Caturegli P: IgG4-related hypophysitis: a new addition to the hypophysitis spectrum. J Clin Endocrinol Metab 96:1971-1980, 2011

17. Leung GK, Lopes MB, Thorner MO, Vance ML, Laws ER, Jr.: Primary hypophysitis: a single-center experience in 16 cases. J Neurosurg 101:262-271, 2004

18. Levine SN, Benzel EC, Fowler MR, Shroyer JV, 3rd, Mirfakhraee M: Lymphocytic adenohypophysitis: clinical, radiological, and magnetic resonance imaging characterization. Neurosurgery 22: 937-941, 1988

19. McIntyre EA, Perros P: Fatal inflammatory hypophysitis. Pituitary 10:107-111, 2007

20. Pamir MN, Zirh TA, Ozek MM, Sav A, Erzen C, Erbengi T: Magnetic resonance imaging in the diagnosis of idiopathic giantcell granulomatous hypophysitis: a rare cause of hyperprolactinaemia. Neurochirurgia (Stuttg) 36:20-25, 1993

21. Sato N, Sze G, Endo K: Hypophysitis: endocrinologic and dynamic MR findings. AJNR Am J Neuroradiol 19:439-444, 1998 
22. Sautner D, Saeger W, Ludecke DK, Jansen V, Puchner MJ: Hypophysitis in surgical and autoptical specimens. Acta Neuropathol 90:637-644, 1995

23. Stott V, Manning P, Hung N: Idiopathic granulomatous hypophysitis. N Z Med J 118:U1355, 2005

24. Su SB, Zhang DJ, Yue SY, Zhang JN: Primary granulomatous hypophysitis: a case report and literature review. Endocr J 58: 467-473, 2011

25. Taylon C, Duff TA: Giant cell granuloma involving the pituitary gland. Case report. J Neurosurg 52:584-587, 1980

26. Thiryayi W, Donaldson MH, Border D, Tyagi A: An enhancing pituitary lesion in a young woman: a diagnostic dilemma. J Clin
Neurosci 14:286-288, 2007

27. Torino F, Barnabei A, De Vecchis L, Salvatori R, Corsello SM: Hypophysitis induced by monoclonal antibodies to cytotoxic $\mathrm{T}$ lymphocyte antigen 4: challenges from a new cause of a rare disease. Oncologist 17:525-535, 2012

28. Vasile M, Marsot-Dupuch K, Kujas M, Brunereau L, Bouchard P, Comoy J, et al.: Idiopathic granulomatous hypophysitis: clinical and imaging features. Neuroradiology 39:7-11, 1997

29. Yoshioka M, Yamakawa N, Saito H, Yoneda M, Nakayama T, Kuroki M, et al.: Granulomatous hypophysitis with meningitis and hypopituitarism. Intern Med 31:1147-1150, 1992 